\title{
Barreras asociadas a la adherencia al tratamiento de tuberculosis en Cali y Buenaventura, Colombia, 2012*
}

\section{Obstacles Associated with the Adherence to Tuberculosis Treatment in Cali and Buenaventura, Colombia, 2012}

\section{Barreiras associadas à aderência ao tratamento da tuberculose em Cali e Buenaventura, Colômbia, 2012}

Fecha de recepción: 22-09-2016 Fecha de aceptación: 15-03-2017 Disponible en línea: 30-05-2017 doi:10.11144/Javeriana.rgps16-32.baat

Cómo citar este artículo:

Carvajal-Barona R, Tovar-Cuevas LM, Aristizábal-Grisales JC, Varela-Arévalo MT. Barreras asociadas a la adherencia al tratamiento de tuberculosis en Cali y Buenaventura, Colombia, 2012. Rev Gerenc Polít Salud. 2017; 16 (32): 68-84. https://doi.org/10.11144/Javeriana.rgps16-32.baat

\author{
Rocío Carvajal-Barona** \\ Luis Miguel Tovar-Cuevas*** \\ Juan Carlos Aristizábal-Grisales**** \\ María Teresa Varela-Arévalo ${ }^{* * * * *}$
}

Artículo de investigación, derivado del proyecto Barreras sociales, administrativas y culturales que se presentan en los pacientes con diagnóstico de TB para un manejo integral con éxito en el tratamiento acortado estrictamente supervisado para tuberculosis en el municipio de Cali y Buenaventura. Investigación financiada por el Fondo Mundial de lucha contra el SIDA, la tuberculosis y la malaria y por la Organización Internacional para las Migraciones de Colombia. Contrato número: PSPJ 1693-2012.

** Enfermera, magíster en Salud Pública, investigadora del Grupo Salud y Calidad de Vida, Pontificia Universidad Javeriana, Cali. Correo electrónico: rcarvajal@javerianacali.edu.co

*** Economista, magíster en Salud Pública, profesor del Departamento de Economía, Facultad de Ciencias Económicas y Administrativas, e investigador de los grupos Salud y Calidad de Vida y Economía, Gestión y Salud, Pontificia Universidad Javeriana, Cali. Correspondencia: Luis Miguel Tovar Cuevas. Calle 18 No. 118 -250, Departamento de Economía, Pontificia Universidad Javeriana Cali. Correo electrónico: Itovar@javerianacali.edu.co luismtovar@ gmail.com

**** Estadístico, magíster en Epidemiología, profesor del Departamento de Salud Pública y Epidemiología, Facultad Ciencias de la Salud, e investigador del Grupo Economía, Gestión y Salud, Pontificia Universidad Javeriana, Cali. Correo electrónico: jcaristizabal@javerianacali.edu.co

***** Psicóloga, magíster en Epidemiología, profesora del Departamento de Ciencias Sociales, Facultad de Humanidades y Ciencias Sociales, e investigadora del Grupo Salud y Calidad de Vida, Pontificia Universidad Javeriana, Cali. Correo electrónico: mtvarela@javerianacali.edu.co 


\section{Resumen}

Objetivo: determinar barreras asociadas a la adherencia al tratamiento acortado, estrictamente supervisado de tuberculosis (TB) en dos municipios de Colombia. Métodos: estudio de casos (128) y controles (182). Se realizó análisis univariado, bivariado y regresión logística. Resultados: los factores asociados a la no adherencia al tratamiento de TB son: no convivir con familiares $(\mathrm{OR}=4.54$; IC95\% 1.45-14.22), inasistencia a reuniones religiosas $(\mathrm{OR}=28.17$; IC95\% 4.37-181.75), no presentar antecedentes de manejo para una enfermedad mayor a dos semanas (OR=3.37; IC95\% 1.59-7.13), no reconocer que el tratamiento de TB dura hasta seis meses (OR=3.51; IC95\% 1.87-6.59), consumir drogas (OR=3.41; IC95\% 0.95-12.23), falta de suministro ocasional del tratamiento por parte de los servicios de salud (OR=5.53; IC95\% 1.20-5.52). Conclusiones: el diseño y desarrollo de estrategias para asegurar la adherencia al tratamiento de TB debe tener en cuenta, además de los factores individuales comportamentales de los pacientes, los factores sociales y administrativos que afectan su éxito.

Palabras clave: tuberculosis; adherencia al tratamiento; Cali, Buenaventura; Colombia.

\section{Abstract}

Objective: to determine obstacles associated with the adherence to the strictly supervised treatment of tuberculosis (TB) in two municipalities of Colombia. Methods: case studies (128) and controls (182). Univariate, bivariate, and logistic regression analyzes were performed. Results: the factors associated with nonadherence to TB treatment were: no cohabitation with relatives $(\mathrm{OR}=4.54,95 \% \mathrm{CI} 1.45-14.22)$, non-attendance to religious meetings $(\mathrm{OR}=28.17,95 \% \mathrm{CI}$ 4.37-181.75), no antecedents regarding dealing with an illness lasting over two weeks $(\mathrm{OR}=$ $3.37,95 \%$ CI 1.59-7.13), did not recognize that $\mathrm{TB}$ treatment lasts for up to six months $(\mathrm{OR}=$ $3.51,95 \%$ CI 1.87-6.59), drug use (OR $=3.41,95 \%$ CI 0.95-12.23), occasional lack of treatment supplies by the health services $(\mathrm{OR}=5.53,95 \% \mathrm{CI} 1.20-5.52)$. Conclusions: The design and development of strategies to ensure adherence to TB treatment must take into account, in addition to the individual behavioral factors of patients, the social and administrative factors that affect their success.

Keywords: tuberculosis; medication adherence; Cali, Buenaventura; Colombia.

\section{Resumo}

Objetivo: determinar barreiras associadas à aderência ao tratamento recortado, rigorosamente supervisionado de tuberculose (TB) em dois municípios da Colômbia. Métodos: estudo de casos (128) e controlos (182). Análise univariada, bivariada e regressão logística foram feitas. Resultados: os fatores associados à não aderência ao tratamento da TB são: não conviver com familiares ( $\mathrm{OR}=4.54$; IC95 \% 1.45-14.22), não comparecimento a reuniões religiosas ( $\mathrm{OR}=28.17$; IC95 \% 4.37-181.75), não apresentar antecedentes de manejo para doenças maiores do que duas semanas $(\mathrm{OR}=3.37$; IC95\% 1.59-7.13), não reconhecer que o tratamento de TB dura até seis meses (OR=3.51; IC95\% 1.87-6.59), consumir drogas (OR=3.41; IC95\% 0.95-12.23), falta de fornecimento ocasional do tratamento por parte dos serviços de saúde (OR=5.53; IC95\% 1.20-5.52). Conclusões: a concepção e desenvolvimento de estratégias para garantir à adesão ao tratamento da TB deve levar em conta, além dos fatores individuais comportamentais dos pacientes, os fatores sociais e administrativos que afetam seu sucesso.

Palavras chave: tuberculose; adesão ao tratamento; Cali; Buenaventura; Colômbia 


\section{Introducción}

De acuerdo con la Organización Mundial de la Salud (OMS), en el año 2015 se alcanzó la meta de frenar y revertir la incidencia de la tuberculosis (TB) que se había planteado en el año 2000 en los Objetivos de Desarrollo del Milenio y se redujo la prevalencia y mortalidad por dicha enfermedad. Sin embargo, a pesar de estos avances y de que casi todos los casos se pueden curar, la TB sigue siendo una gran amenaza para la salud pública mundial (1). Las barreras al acceso al tratamiento para esta enfermedad, así como la no adherencia o abandono del tratamiento representan un gran problema para su control, toda vez que son la principal causa de recaída de los pacientes y generan complicaciones como la drogorresistencia, que contribuye al incremento de la prevalencia de la infección $(2,3)$.

Investigaciones en el mundo sobre no adherencia o abandono del tratamiento anti-TB han podido establecer que estos factores incrementan la morbilidad y mortalidad de los pacientes, disminuyen la tasa de curación, elevan la transmisión poblacional, aumentan el número de enfermos crónicos, posibilitan la aparición de tuberculosis multirresistente a drogas-MDR-TB y aumentan los costos del tratamiento (4 -7), igualmente han podido establecer que la no adherencia y abandono del tratamiento están determinados por factores psicosociales, sociodemográficos, económicos, culturales y de los servicios de salud $(4,5,8-13)$.

Cali y Buenaventura son dos ciudades principales del departamento del Valle del Cauca, uno de los más densamente poblados de Colombia, que aporta el $11 \%$ de los casos de TB del país y donde la mayoría de sus habitantes reside en el área urbana $(86.4 \%)$. Estas dos ciudades presentan un importante avance económico y social, aunque en ellas confluyen fenómenos sociales como la violencia, el desplazamiento forzado, las migraciones y la pobreza $(14,15)$. Adicionalmente, reportan el mayor número de casos de TB del departamento y un porcentaje de abandono del tratamiento de entre 9 y $14 \%$, cifra que se encuentra por encima de los estándares nacionales establecidos para una buena gestión del programa de control de TB. Por otra parte, la MDR y la extremada drogorresistencia (XDR) a medicamentos anti-TB son un problema en el departamento del Valle del Cauca, donde Cali y Buenaventura son los municipios que reportan el $90 \%$ de estos casos (15).

Aunque el Valle del Cauca ha adoptado e implementado la estrategia del tratamiento administrado estrictamente supervisado DOTS-TAES, la no adherencia, la MDR y la XDR siguen siendo un problema. Por tal motivo, este estudio se planteó determinar cuáles son los factores individuales, sociales, administrativos y culturales que se presentan en los pacientes de TB que se convierten en barreras para la adherencia al tratamiento acortado estrictamente supervisado de TB en los municipios de Cali y Buenaventura.

\section{Materiales y métodos}

Tipo de estudio: se diseñó un estudio de casos y controles de acuerdo con las disposiciones éticas nacionales e internacionales para la investigación con humanos (Declaración de Helsinki y Resolución 8430 de 1993 del Ministerio de Salud de Colombia (16)).

Población: expacientes del programa de TB en Cali y Buenaventura en el año 2012. Los criterios de inclusión de las personas que participaron en esta investigación fueron residir en la zona urbana, no poseer ninguna enfermedad mental conocida y/o discapacidad 
que les impidiera responder el cuestionario y aceptar su participación en el estudio voluntariamente a través del diligenciamiento del consentimiento informado.

Muestra y selección: se calculó con base en un $20 \%$ de prevalencia de exposición en los controles a barreras que generan el incumplimiento al tratamiento de TB, confiabilidad del $95 \%$, poder de $80 \%$ y no respuesta de $20 \%$, para una relación de 187 casos y 187 controles. El tamaño de la muestra final fue de 128 casos expacientes no adherentes o abandonadores y 182 controles expacientes que empezaron y terminaron su tratamiento en seis meses, para un total de 310 personas.

Variables: la variable dependiente es una dummy que mide si los individuos se adhieren o no al tratamiento de TB. Esta variable clasifica en la categoría de "adherente-control" a los individuos enfermos con TB que cumplieron el tratamiento con al menos el $90 \%$ de las dosis de tratamiento supervisado. En caso contrario son clasificados como "no adherente o abandonador-caso". Las variables de exposición fueron clasificadas en cuatro categorías: características individuales, administrativas, sociodemográficas y culturales presentes en los pacientes en el momento que recibieron tratamiento para la TB.

\section{Técnicas de recolección de información:} para recolectar la información se construyó un cuestionario estructurado, teniendo en cuenta instrumentos previos que habían sido utilizados para medir adherencia al tratamiento anti TB (17) y los planteamientos del modelo de acceso y uso de servicios de salud de Aday y Andersen (18). El cuestionario fue validado mediante entrevista cognitiva y prueba piloto con el fin de asegurar la comprensión de las preguntas y mejorar la validez de la medición. La aplicación del cuestionario se llevó a cabo en las viviendas o sitios acordados, mediante cita previa, a los expacientes que estuvieron inscritos en los programas de control de TB el año inmediatamente anterior. La variable resultado se midió revisando las tarjetas de control de los medicamentos anti-TB de los entrevistados y clasificándolos como caso o control de acuerdo con el número de dosis recibidas, según los registros. Adicionalmente, se diseñó un consentimiento informado según la legislación de ética colombiana para informar a los participantes el propósito del estudio, procedimientos, riesgos y la participación voluntaria.

La calidad de los datos se controló antes, durante y después de la recolección. Antes de la recolección se desarrollaron manuales de procedimientos, los encuestadores y supervisores participaron de una jornada de capacitación y se realizó prueba piloto de instrumentos y procedimientos. Durante la recolección se hicieron visitas de seguimiento a los equipos operativos para evaluar el cumplimiento de los procesos; las encuestas fueron revisadas diariamente por los supervisores de campo y en caso de errores se devolvieron para corrección. Después de la recolección, los cuestionarios se digitaron en una base de datos diseñada en Epi-info (versión 7.0). Luego se tomó $10 \%$ de las encuestas realizadas y se comparó con los registros de la base de datos.

Análisis de datos: los datos fueron analizados con el programa estadístico SPSS18. Una vez realizada la limpieza y depuración de la base de datos, se llevó a cabo un análisis exploratorio. Posteriormente, para identificar factores que determinan el acceso al tratamiento anti-TB se construyó la variable dependiente dicotómica tratamiento anti-TB y se realizó análisis bivariados para identificar la asociación de las variables de exposición con la variable resultado. Después, con las asociaciones que presentaron probabilidades 
menores de 0.20 y tamaños de muestra suficientes, se construyó un modelo de regresión logística múltiple. La selección de variables del modelo final se realizó utilizando el método de eliminación de variables hacia atrás Backward, con una probabilidad de retiro de 0.20. Una vez obtenido el modelo, se hizo el diagnóstico y la evaluación de su ajuste, a través de la precisión general, la bondad de ajuste a través de la prueba Hosmer y Lemeshow, la prueba omnibus sobre los coeficientes del modelo y la presencia de colinearidad, mediante la matriz de correlaciones de Spearman.

\section{Resultados}

\section{Estadísticas descriptivas}

En el estudio participaron 310 sujetos, de los cuales $48 \%$ eran de Cali y $52 \%$ de Buenaventura. $58.7 \%$ de ellos fueron adherentes (controles) y $41.3 \%$ no adherentes-abandonadores (casos). En esta investigación se analizaron por lo menos sesenta variables que representan características individuales, administrativas, sociodemográficas y culturales presentes en los pacientes en el momento que recibieron tratamiento para la TB. Sin embargo, dada la extensión de estos resultados, en este artículo solo se presentan cerca de la mitad de las variables consideradas, pues se entiende que son las más relevantes de acuerdo con la literatura revisada. Se reportan todas las variables del estudio que mostraron asociación significativa a un nivel de significancia del $5 \%$.

\section{Características individuales y culturales} (véase tabla 1): al momento del tratamiento de TB, $89.7 \%$ de los encuestados informaron que vivían con su familia, y con respecto a su relación con esta, $85.2 \%$ respondieron que era buena, $9.7 \%$ regular y $3.2 \%$ mala. $\mathrm{Al}$ indagar si habían sufrido alguna enfermedad que hubiera requerido un tratamiento por más de dos semanas, $28.4 \%$ manifestaron que sí se había presentado esta situación. Adicionalmente, con relación a fumar, consumo de drogas y alcohol, los encuestados informaron que sí tenían estos hábitos en $14.5,8.7$ y $16.5 \%$, respectivamente. Del total de los entrevistados que manifestaron conocer alguna persona con TB, $70.5 \%$ dijeron haber tenido algún tipo de contacto con estas personas.

$\mathrm{Al}$ indagar acerca del conocimiento de la duración del tratamiento, se encontró que este tiempo medido en meses fue muy variable, entre 1 y 312 meses; tres de cada cuatro de los encuestados manifestaron que la duración de su tratamiento fue menor a doce meses; cuando se preguntó por la credibilidad en el tratamiento que habían recibido o estaban recibiendo $95.2 \%$ de los encuestados manifestó que confiaban en la efectividad; durante el tratamiento de TB $26.1 \%$ de las personas informaron que se les había presentado algún tipo de complicación. Del total de las personas que manifestaron haber presentado algún tipo de complicación, $70 \%$ manifestaron que fue necesario, debido a esa complicación, dejar de realizar sus actividades normales como trabajar o estudiar, entre otras. Por otro lado, en el aspecto cultural, $48.3 \%$ del total de los participantes en el estudio informaron asistir diaria o semanalmente a algún grupo religioso. 
Tabla 1. Características individuales y Culturales (total, CONTROles y Casos)

\begin{tabular}{|c|c|c|c|c|c|c|c|}
\hline \multirow{2}{*}{ Pregunta/característica } & \multirow{2}{*}{$\begin{array}{l}\text { Opciones } \\
\text { indicador }\end{array}$} & \multicolumn{2}{|c|}{ Total } & \multicolumn{2}{|c|}{ Controles } & \multicolumn{2}{|c|}{ Casos } \\
\hline & & $\mathbf{n}$ & $\%$ & $\mathbf{n}$ & $\%$ & $\mathbf{n}$ & $\%$ \\
\hline \multirow{2}{*}{$\begin{array}{l}\text { Al momento del trata- } \\
\text { miento, vivía usted con } \\
\text { su familia }\end{array}$} & No & 27 & 8.7 & 11 & 6.1 & 16 & 12.8 \\
\hline & Sí & 278 & 89.7 & 169 & 93.9 & 109 & 87.2 \\
\hline \multirow{3}{*}{$\begin{array}{l}\text { Durante el tratamiento } \\
\text { de } \mathrm{TB} \text {, las relaciones } \\
\text { familiares eran }\end{array}$} & Buenas & 264 & 85.2 & 167 & 92.3 & 97 & 78.9 \\
\hline & Regulares & 30 & 9.7 & 12 & 6.6 & 18 & 14.6 \\
\hline & Malas & 10 & 3.2 & 2 & 1.1 & 8 & 6.5 \\
\hline \multirow{2}{*}{$\begin{array}{l}\text { Fue tratado por otra } \\
\text { enfermedad por más de } \\
\text { dos semanas }\end{array}$} & No & 217 & 70.0 & 120 & 66.7 & 97 & 77.6 \\
\hline & Sí & 88 & 28.4 & 60 & 33.3 & 28 & 22.4 \\
\hline \multirow{2}{*}{ Consumo de cigarrillo } & No & 261 & 84.2 & 162 & 89.5 & 99 & 79.2 \\
\hline & Sí & 45 & 14.5 & 19 & 10.5 & 26 & 20.8 \\
\hline \multirow{2}{*}{$\begin{array}{l}\text { Consume drogas como } \\
\text { marihuana, bazuco, } \\
\text { cocaína u otras }\end{array}$} & No & 279 & 90.0 & 174 & 96.1 & 105 & 84.0 \\
\hline & Sí & 27 & 8.7 & 7 & 3.9 & 20 & 16.0 \\
\hline \multirow{2}{*}{$\begin{array}{l}\text { Consume alcohol con } \\
\text { frecuencia }\end{array}$} & No & 255 & 82.3 & 156 & 86.2 & 99 & 79.2 \\
\hline & Sí & 51 & 16.5 & 25 & 13.8 & 26 & 20.8 \\
\hline \multirow{2}{*}{$\begin{array}{l}\text { Ha tenido contacto } \\
\text { con personas enfermas } \\
\text { de TB }\end{array}$} & No & 46 & 29.5 & 21 & 22.6 & 25 & 39.7 \\
\hline & Sí & 110 & 70.5 & 72 & 77.4 & 38 & 60.3 \\
\hline \multirow{3}{*}{$\begin{array}{l}\text { Cuánto tiempo dura } \\
\text { el tratamiento de TB } \\
\text { (meses) }\end{array}$} & Mínimo & 1 & & 1 & & 1 & \\
\hline & Máximo & 312 & & 180 & & 312 & \\
\hline & Cuartil 3 & 12 & & 12 & & 24 & \\
\hline \multirow{2}{*}{$\begin{array}{l}\text { Cree en la efectividad } \\
\text { del tratamiento anti-TB }\end{array}$} & No & 10 & 3.2 & 2 & 1.1 & 8 & 6.3 \\
\hline & Sí & 295 & 95.2 & 177 & 98.9 & 118 & 93.7 \\
\hline \multirow{2}{*}{$\begin{array}{l}\text { Se le presentó algún } \\
\text { tipo de complicación } \\
\text { durante el tratamiento } \\
\text { de TB }\end{array}$} & No & 224 & 72.3 & 142 & 78.5 & 82 & 66.1 \\
\hline & Sí & 81 & 26.1 & 39 & 21.5 & 42 & 33.9 \\
\hline \multirow{2}{*}{$\begin{array}{l}\text { Fue necesario, debido } \\
\text { a una complicación en } \\
\text { el tratamiento, dejar de } \\
\text { realizar sus actividades } \\
\text { normales }\end{array}$} & No & 24 & 30.0 & 15 & 39.5 & 9 & 21.4 \\
\hline & Sí & 56 & 70.0 & 23 & 60.5 & 33 & 78.6 \\
\hline \multirow{2}{*}{$\begin{array}{l}\text { Usted tiene alguna } \\
\text { creencia religiosa o } \\
\text { espiritual }\end{array}$} & No & 43 & 13.9 & 17 & 11 & 7 & 6.4 \\
\hline & Sí & 263 & 84.8 & 68 & 44.2 & 35 & 32.1 \\
\hline
\end{tabular}

Fuente: elaboración propia. 


\section{Rocío Carvajal-Barona - LUIS Miguel Tovar-Cuevas •

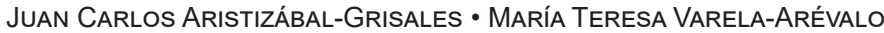

Características administrativas (véase tabla 2): al momento del tratamiento de TB, el $86 \%$ estaban afiliados al sistema de salud; la mayoría pertenecía al régimen subsidiado $(66.5 \%)$. Al indagar por problemas en el suministro de tratamiento de TB por parte de la institución de salud que lo suministraba, $5.2 \%$ de los encuestados informaron que se había presentado esta situación. Y con relación a la posibilidad de asistir al tratamiento en el horario propuesto por la institución de salud, $3.2 \%$ del total manifestó que no le fue posible asistir en el horario propuesto.

Tabla 2. Características administrativas (total, CONTROLES y Casos)

\begin{tabular}{|c|c|c|c|c|c|c|c|}
\hline \multirow{2}{*}{ Pregunta/característica } & \multirow{2}{*}{$\begin{array}{l}\text { Opciones/ } \\
\text { indicador }\end{array}$} & \multicolumn{2}{|c|}{ Total } & \multicolumn{2}{|c|}{ Controles } & \multicolumn{2}{|c|}{ Casos } \\
\hline & & $\mathbf{n}$ & $\%$ & $\mathbf{n}$ & $\%$ & $\mathbf{n}$ & $\%$ \\
\hline \multirow{3}{*}{$\begin{array}{l}\text { Tipo de afiliación } \\
\text { a salud }\end{array}$} & Contributivo & 57 & 18.4 & 43 & 23.8 & 14 & 11.2 \\
\hline & Subsidiado & 206 & 66.5 & 119 & 65.7 & 87 & 69.6 \\
\hline & No afiliado & 43 & 13.9 & 19 & 10.5 & 24 & 19.2 \\
\hline \multirow{2}{*}{$\begin{array}{l}\text { Tuvo problemas con el } \\
\text { proveedor de servicios } \\
\text { de salud en el suminis- } \\
\text { tro del tratamiento }\end{array}$} & No & 290 & 93.5 & 176 & 97.2 & 114 & 91.2 \\
\hline & Sí & 16 & 5.2 & 5 & 2.8 & 11 & 8.8 \\
\hline \multirow{2}{*}{$\begin{array}{l}\text { Facilidad para asistir al } \\
\text { tratamiento de TB en el } \\
\text { horario propuesto por } \\
\text { la institución de salud }\end{array}$} & No & 10 & 3.2 & 3 & 1.7 & 7 & 5.6 \\
\hline & Sí & 292 & 94.2 & 174 & 98.3 & 118 & 94.4 \\
\hline
\end{tabular}

Fuente: elaboración propia

Características sociodemográficas (véase tabla 3): la edad de los participantes en esta investigación presentó un promedio de 43.9 años y desviación estándar de 18.6 años, con un rango de edad bastante amplio, entre 15 y 99 años (coeficiente de variación 42.3\%). Con relación a la etnia, $43.2 \%$ se consideraban afrodescendientes y $31 \%$ mestizos. El $43.8 \%$ informó que tenía pareja estable (unión libre o casado) y el $54 \%$ sostuvo que se encontraba sin pareja estable (solteros, viudos, separados o divorciados). En cuanto al nivel educativo, 9.4\% del total de los participantes manifestó no tener ningún nivel de educación, $80 \%$ con nivel educativo entre primaria y secundaria y solo $9 \%$ en nivel de educación superior o postgrado. Con relación a la actividad realizada durante el tratamiento de TB, 38.1\% realizó oficios relacionados con el hogar, $36.1 \%$ informó estar trabajando, 5.8\% trabajó y realizó oficios del hogar conjuntamente y $4.8 \%$ se encontraba estudiando, el resto de los encuestados manifestó que se encontraba buscando trabajo o en el desarrollo de otras actividades.

Con respecto a los ingresos económicos mensuales, aproximadamente una cuarta parte $(23 \%)$ de los participantes manifestó tener ingresos por debajo de $\$ 200000$ pesos, $25 \%$ entre $\$ 201000$ y $\$ 500000$ y 36\% señalaron que "no sabían-no respondieron" esta pregunta. Al indagar por la posibilidad de contar con el dinero suficiente para el desplazamiento hasta la institución de salud para recibir el tratamiento, $50.6 \%$ del total de los encuestados manifestó no disponer de estos recursos. Del total de personas que participaron en este estudio, 94.8\% manifestó que su lugar de residencia se encontraba en zona urbana. 
Tabla 3. Características sociodemográficas (total, CONTROles y Casos)

\begin{tabular}{|c|c|c|c|c|c|c|c|}
\hline \multirow{2}{*}{ Pregunta/característica } & \multirow{2}{*}{ Opciones/indicador } & \multicolumn{2}{|c|}{ Total } & \multicolumn{2}{|c|}{ Controles } & \multicolumn{2}{|c|}{ Casos } \\
\hline & & n & $\%$ & $\mathbf{n}$ & $\%$ & $\mathbf{n}$ & $\%$ \\
\hline \multirow{4}{*}{ Edad } & Promedio & 43.91 & & 44.99 & & 42.37 & \\
\hline & Desv. estándar & 18.56 & & 19.45 & & 17.15 & \\
\hline & Mínimo & 15 & & 15 & & 15 & \\
\hline & Máximo & 99 & & 99 & & 85 & \\
\hline \multirow{5}{*}{ Etnia } & Blanco(a) & 50 & 16.1 & 26 & 14.3 & 24 & 18.9 \\
\hline & Negro(a)/afrodescendiente & 134 & 43.2 & 79 & 43.4 & 55 & 43.3 \\
\hline & Mestizo(a) & 96 & 31.0 & 61 & 33.5 & 35 & 27.6 \\
\hline & Indígena & 14 & 4.5 & 9 & 4.9 & 5 & 3.9 \\
\hline & Otro & 15 & 4.8 & 7 & 3.8 & 8 & 6.3 \\
\hline \multirow{5}{*}{ Estado civil } & Soltero(a) & 130 & 41.9 & 61 & 33.9 & 69 & 55.2 \\
\hline & Casado(a) & 41 & 13.2 & 30 & 16.7 & 11 & 8.8 \\
\hline & Vivía(e) en unión libre & 95 & 30.6 & 59 & 32.8 & 36 & 28.8 \\
\hline & Viudo(a) & 23 & 7.4 & 17 & 9.4 & 6 & 4.8 \\
\hline & Separado(a)/divorciado(a) & 16 & 5.2 & 13 & 7.2 & 3 & 2.4 \\
\hline \multirow{5}{*}{ Nivel educativo } & Ninguna & 29 & 9.4 & 16 & 8.8 & 13 & 10.5 \\
\hline & Primaria & 106 & 34.2 & 62 & 34.3 & 44 & 35.5 \\
\hline & Secundaria & 143 & 46.1 & 85 & 47.0 & 58 & 46.8 \\
\hline & Superior & 24 & 7.7 & 15 & 8.3 & 9 & 7.3 \\
\hline & Postgrado & 3 & 1.0 & 3 & 1.7 & & \\
\hline \multirow{6}{*}{$\begin{array}{l}\text { Actividad realizada } \\
\text { (ocupación) }\end{array}$} & Trabajo & 112 & 36.1 & 57 & 31.7 & 55 & 44.0 \\
\hline & Oficios del hogar & 118 & 38.1 & 78 & 43.3 & 40 & 32.0 \\
\hline & Estudio & 15 & 4.8 & 11 & 6.1 & 4 & 3.2 \\
\hline & Trabajo y oficios del hogar & 18 & 5.8 & 13 & 7.2 & 5 & 4.0 \\
\hline & Buscó trabajo & 17 & 5.5 & 9 & 5.0 & 8 & 6.4 \\
\hline & Otra actividad & 25 & 8.1 & 12 & 6.7 & 13 & 10.4 \\
\hline \multirow{5}{*}{ Ingresos económicos } & Menos o igual a 100000 & 29 & 9.4 & 18 & 9.9 & 11 & 8.8 \\
\hline & Entre 101000 y 200000 & 40 & 12.9 & 24 & 13.3 & 16 & 12.8 \\
\hline & Entre 201000 y 500000 & 79 & 25.5 & 54 & 29.8 & 25 & 20.0 \\
\hline & Entre 501000 y 1000000 & 34 & 11.0 & 25 & 13.8 & 9 & 7.2 \\
\hline & Más de 1500000 & 8 & 2.6 & 6 & 3.3 & 2 & 1.6 \\
\hline \multirow{2}{*}{$\begin{array}{l}\text { Cuenta con dinero sufi- } \\
\text { ciente para el desplaza- } \\
\text { miento hasta la institución } \\
\text { de salud }\end{array}$} & No & 157 & 50.6 & 77 & 42.5 & 80 & 64.0 \\
\hline & Sí & 149 & 48.1 & 104 & 57.5 & 45 & 36.0 \\
\hline \multirow{2}{*}{ Área de residencia } & Urbana & 294 & 94.8 & 176 & 97.8 & 118 & 97.5 \\
\hline & Rural & 7 & 2.3 & 4 & 2.2 & 3 & 2.5 \\
\hline
\end{tabular}

Fuente: elaboración propia 


\section{Rocío CARVAJAL-BARONA • LUIS MIGUEL TOVAR-CuEVAS •

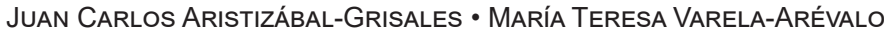

\section{Análisis bivariado}

Si se tiene en cuenta el modelo de uso de los servicios de salud de Aday y Andersen (18), las variables consideradas en este estudio fueron reorganizadas en grupos que corresponden a aspectos o factores que se encuentran relacionados con la adherencia al tratamiento de TB. Entre dichos aspectos están los siguientes:

1. Características de la población en situación de riesgo: factores de predisposición (edad, etnia, estado civil, nivel educativo, haber recibido tratamiento para otra enfermedad por más de dos semanas), red de apoyo social (relaciones familiares, vivir con la familia, asistencia a grupo religioso), antecedentes personales (consumo de cigarrillo, consumo de drogas, consumo de alcohol), factores de capacidad (tipo de afiliación a salud, ingreso, área de residencia, ocupación, recursos para asumir costos de transporte).

2. Satisfacción de las necesidades del consumidor: antecedentes de complicaciones con el tratamiento de TB, reporte de afectación de actividades normales por causa del tratamiento, conocimientos y percepción del tratamiento anti-TB (contacto con personas con TB, conocimiento de la duración del tratamiento y creencias sobre la efectividad de este).

3. Características del sistema de prestación de servicios de salud: falta de suministro ocasional del tratamiento, facilidad de horarios de las citas.

En la tabla 4 se reportan los resultados estadísticamente significativos del análisis bivariado.

Tabla 4. Resultados estadísticamente Significativos del anÁLISIS bivariado

\begin{tabular}{|c|c|c|c|c|c|}
\hline \multirow{2}{*}{\multicolumn{2}{|c|}{ Variable }} & \multirow{2}{*}{$\mathbf{p}$} & \multirow{2}{*}{$\begin{array}{l}\text { OR } \\
\text { crudo }\end{array}$} & \multicolumn{2}{|c|}{ IC95\% } \\
\hline & & & & Linf & Lsup \\
\hline \multicolumn{6}{|c|}{ Características de la población en situación de riesgo } \\
\hline \multicolumn{6}{|l|}{ Factores de predisposición } \\
\hline \multirow{5}{*}{ Estado civil } & Soltero(a) & & 1 & & \\
\hline & Casado(a) & 0.004 & $\underline{0.32}$ & 0.15 & 0.70 \\
\hline & Vivía( e) en unión libre & 0.025 & $\underline{0.54}$ & 0.32 & 0.93 \\
\hline & Viudo(a) & 0.021 & $\underline{0.31}$ & 0.12 & 0.84 \\
\hline & $\begin{array}{l}\text { Separado(a)/Divorcia- } \\
\text { do(a) }\end{array}$ & 0.017 & $\underline{\mathbf{0 . 2 0}}$ & 0.06 & 0.75 \\
\hline \multirow{2}{*}{$\begin{array}{l}\text { Fue tratado por otra enfermedad } \\
\text { por más de dos semanas }\end{array}$} & Sí & & 1 & & \\
\hline & No & 0.039 & $\underline{1.73}$ & 1.03 & 2.92 \\
\hline \multicolumn{6}{|l|}{ Red de apoyo social } \\
\hline \multirow{2}{*}{$\begin{array}{l}\text { Al momento del tratamiento de TB } \\
\text { vivía usted con su familia }\end{array}$} & Sí & & 1 & & \\
\hline & No & 0.048 & $\underline{2.26}$ & 1.01 & 5.04 \\
\hline \multirow{3}{*}{$\begin{array}{l}\text { Durante el tratamiento de TB las } \\
\text { relaciones familiares eran }\end{array}$} & Malas & & 1 & & \\
\hline & Buenas & 0.016 & $\underline{0.15}$ & 0.03 & 0.70 \\
\hline & Regulares & 0.262 & 0.38 & 0.07 & 2.08 \\
\hline
\end{tabular}




\begin{tabular}{|c|c|c|c|c|c|}
\hline \multirow{5}{*}{ Asiste a grupos religiosos } & Diariamente & & 1 & & \\
\hline & Semanalmente & 0.652 & 1.25 & 0.47 & 3.30 \\
\hline & Mensualmente & 0.394 & 1.54 & 0.57 & 4.13 \\
\hline & Cada año & 0.174 & 2.16 & 0.71 & 6.54 \\
\hline & Nunca va a misa o culto & 0.000 & $\underline{24.29}$ & 4.44 & 132.84 \\
\hline \multicolumn{6}{|l|}{ Antecedentes personales } \\
\hline \multirow{2}{*}{ Consumo de cigarrillo } & No & & 1 & & \\
\hline & Sí & 0.014 & 2.24 & 1.18 & 4.26 \\
\hline \multirow{2}{*}{ Consumo de drogas } & No & & 1 & & \\
\hline & Sí & 0.001 & $\underline{4.73}$ & 1.94 & 11.58 \\
\hline \multicolumn{6}{|l|}{ Factores de capacidad } \\
\hline \multirow{3}{*}{$\begin{array}{l}\text { Tipo de afiliación a servicios de } \\
\text { salud }\end{array}$} & Contributivo & & 1 & & \\
\hline & Subsidiado & 0.017 & $\underline{2.25}$ & 1.16 & 4.36 \\
\hline & No afiliado & 0.002 & $\underline{3.88}$ & 1.65 & 9.10 \\
\hline \multirow{6}{*}{ Actividad realizada (ocupación) } & Trabajo & & 1 & & \\
\hline & Oficios del hogar & 0.020 & $\underline{0.53}$ & 0.31 & 0.90 \\
\hline & Estudio & 0.112 & 0.38 & 0.11 & 1.25 \\
\hline & Trabajo y oficio del hogar & 0.100 & 0.4 & 0.13 & 1.19 \\
\hline & Buscó trabajo & 0.875 & 0.92 & 0.33 & 2.56 \\
\hline & Otra actividad & 0.794 & 1.12 & 0.47 & 2.67 \\
\hline \multirow{2}{*}{$\begin{array}{l}\text { Tuvo dinero suficiente para el des- } \\
\text { plazamiento hasta la institución de } \\
\text { salud donde recibía el tratamiento }\end{array}$} & Sí & & 1 & & \\
\hline & No & 0.006 & $\underline{2.82}$ & 1.34 & 5.93 \\
\hline \multicolumn{6}{|c|}{ Satisfacción de las necesidades del consumidor } \\
\hline \multirow{2}{*}{$\begin{array}{l}\text { Se le presentó algún tipo de compli- } \\
\text { cación durante el tratamiento de } \\
\text { TB }\end{array}$} & No & & 1 & & \\
\hline & Sí & 0.017 & $\underline{1.86}$ & 1.12 & 3.12 \\
\hline \multicolumn{6}{|c|}{ Conocimiento y percepción del tratamiento anti-TB } \\
\hline \multirow{2}{*}{$\begin{array}{l}\text { Contacto con personas enfermas } \\
\text { de TB }\end{array}$} & Sí & & 1 & & \\
\hline & No & 0.023 & $\underline{2.26}$ & 1.12 & 4.55 \\
\hline \multirow{2}{*}{ Cuánto dura el tratamiento de TB } & Hasta seis meses & & 1 & & \\
\hline & Más de seis meses & 0.001 & 2.19 & 1.36 & 3.52 \\
\hline \multirow{2}{*}{$\begin{array}{l}\text { Cree en la efectividad del trata- } \\
\text { miento anti-TB }\end{array}$} & Sí & & 1 & & \\
\hline & No & 0.025 & 6.00 & 1.25 & 28.75 \\
\hline \multicolumn{6}{|c|}{ Características sistema de prestación de servicios de salud } \\
\hline \multirow{2}{*}{$\begin{array}{l}\text { Tuvo problemas con el proveedor } \\
\text { de servicios de salud en el suminis- } \\
\text { tro del tratamiento }\end{array}$} & No & & 1 & & \\
\hline & Sí & 0.027 & $\underline{\mathbf{3 . 4 0}}$ & 1.15 & 10.03 \\
\hline
\end{tabular}

Fuente: elaboración propia 


\section{Características de la población en situación} de riesgo: entre los "factores de predisposición" únicamente el estado civil y tener antecedentes de haber recibido tratamiento para otra enfermedad por más de dos semanas presentaron relación significativa con la adherencia. Se observa que el estar soltero representa una mayor oportunidad de no ser adherente al tratamiento anti-TB, de tal manera que la oportunidad de ser no adherente con respecto a los solteros es $68 \%$ menos entre los casados, $46 \%$ menos en los que viven en unión libre, $69 \%$ menos en los viudos y $80 \%$ menos en los separados/divorciados. En el caso de los pacientes que no tenían antecedentes de haber recibido tratamiento para otra enfermedad por más de dos semanas, la oportunidad de incumplir el tratamiento de TB era 1.73 veces mayor, en comparación con los que sí tenían dicho antecedente.

En el grupo de variables relacionadas con la "red de apoyo social", se encontró mayor oportunidad de incumplimiento del tratamiento entre los pacientes que no viven con su familia (OR = 2.26; IC $95 \% 1.01$ - 5.04). Además, se observó que tener buenas relaciones con los familiares es un factor protector, puesto que reduce la oportunidad de incumplimiento del tratamiento en $85 \%$. Se destaca que los pacientes que nunca asisten a grupos religiosos presentaron una alta oportunidad de incumplimiento del tratamiento, en comparación con quienes asisten diariamente o casi diariamente $(\mathrm{OR}=24.29$; IC $95 \%$ 4.44-132.84). Adicionalmente, entre los pacientes que no han sufrido alguna enfermedad que haya requerido tratamiento por más de dos semanas se presentó una oportunidad de incumplimiento $73 \%$ mayor $(\mathrm{OR}=1.73$; IC $95 \%$ 1.03-2.92) comparada con los que sí presentaron dicho evento.

Respecto a los "antecedentes personales", los pacientes que reportan consumo de cigarrillo y de sustancias alucinógenas presentan, respectivamente, una oportunidad 1.24 y 3.73 veces mayor de incumplir el tratamiento, comparados en ambos casos con los que no reportaron consumo.

Los "factores de capacidad" señalan que se presentó una mayor oportunidad de incumplimiento en el tratamiento de TB en pacientes afiliados al régimen subsidiado de salud $(\mathrm{OR}=2.25$; IC $95 \%$ 1.16-1.36) y en los no afiliados al sistema $(\mathrm{OR}=3.88$; IC $95 \%$ 1.65-9.10) en comparación con los afiliados al régimen contributivo de salud. Así mismo, los pacientes que manifestaron dificultades para asumir los costos del transporte presentaron un riesgo. 1,82 veces mayor de incumplir el tratamiento en comparación con los que tuvieron capacidad económica suficiente para hacerlo. Por otro lado, la ocupación de los individuos durante el tratamiento mostro que desarrollar oficios del hogar reduce la oportunidad de incumplimiento del tratamiento en un $47 \%$, en comparación con los que trabajan.

\section{Satisfacción de las necesidades del consumi-} dor: los pacientes que reportaron complicaciones de salud durante el tratamiento de TB presentaron una oportunidad de incumplir el tratamiento $86 \%$ mayor que los pacientes que no reportaron ninguna complicación. Respecto al "conocimiento y percepción del tratamiento anti-TB”, los pacientes que no han tenido contacto con personas enfermas de TB presentaron una oportunidad de incumplimiento del tratamiento 1.26 veces más que los que sí lo han tenido; del mismo modo, las personas que manifestaron desconocimiento de la duración del tratamiento (más de seis meses) presentaron una oportunidad mayor de incumplimiento con relación a los que sí conocen el tiempo que dura el tratamiento (OR $=2.19$; IC $95 \%$ 1.36-3.52); finalmente, los pacientes que no creen que el tratamiento de TB recibido cure la enfermedad presentaron una oportunidad 
cinco veces mayor de incumplir el tratamiento, en comparación con los que creen en la efectividad del tratamiento.

\section{Características del sistema de prestación} de servicios de salud: los pacientes que informaron falta de suministro ocasional del tratamiento por parte del proveedor de salud presentaron una oportunidad de incumplir el tratamiento. 2,4 veces mayor que aquellos que no tuvieron problemas con el suministro.

El análisis bivariado no arrojó asociación estadísticamente significativa entre el incumplimiento del tratamiento de TB y variables como la edad, la etnia, el género, el nivel educativo, el ingreso, el área de residencia, el consumo de alcohol, el reporte de afectación de actividades normales por causa del tratamiento y la facilidad de los horarios de las citas.

\section{Análisis multivariado}

Una vez realizado el proceso para la construcción de un modelo de regresión logística múltiple utilizando el método de eliminación de variables hacia atrás (Backward), con una probabilidad de retiro de 0.20 , se obtuvo el modelo que se presenta en la tabla 5 .

Tabla 5. Factores asociados a la adherencia al tratamiento para la TB en los municipios de Cali y Buenaventura, Colombia $(\mathrm{N}=310)$

\begin{tabular}{|c|c|c|c|c|c|}
\hline \multirow{2}{*}{ Variable } & & \multirow{2}{*}{$\mathbf{P}$} & \multirow{2}{*}{$\begin{array}{c}\text { OR } \\
\text { ajustado }\end{array}$} & \multicolumn{2}{|c|}{ IC $95 \%$} \\
\hline & & & & $L$ inf & L sup \\
\hline \multicolumn{6}{|c|}{ Características de la población en situación de riesgo } \\
\hline \multirow{2}{*}{$\begin{array}{l}\text { Al momento del tratamiento de TB } \\
\text { vivía usted con su familia }\end{array}$} & Sí & & 1 & & \\
\hline & No & 0.009 & $\underline{4.54}$ & 1.45 & 14.22 \\
\hline \multirow{3}{*}{$\begin{array}{l}\text { Durante el tratamiento de TB las } \\
\text { relaciones familiares eran }\end{array}$} & Malas & & 1 & & \\
\hline & Buenas & 0.032 & $\underline{0.08}$ & 0.01 & 0.81 \\
\hline & Regulares & 0.334 & 0.3 & 0.03 & 3.4 \\
\hline \multirow{5}{*}{ Asiste a grupos religiosos } & Diariamente & & 1 & & \\
\hline & Semanalmente & 0.798 & 0.86 & 0.28 & 2.69 \\
\hline & Mensualmente & 0.568 & 1.41 & 0.44 & 4.53 \\
\hline & Cada año & 0.267 & 2.1 & 0.57 & 7.82 \\
\hline & Nunca & 0 & $\underline{28.17}$ & 4.37 & 181.75 \\
\hline \multirow{2}{*}{$\begin{array}{l}\text { Fue tratado por otra enfermedad } \\
\text { por más de dos semanas }\end{array}$} & Sí & & 1 & & \\
\hline & No & 0.001 & $\underline{3.37}$ & 1.59 & 7.13 \\
\hline \multirow{2}{*}{ Consume drogas } & No & & 1 & & \\
\hline & Sí & 0.06 & $\underline{3.41}$ & 0.95 & 12.23 \\
\hline \multirow{3}{*}{ Tipo de afiliación a salud } & Reg. contributivo & & 1 & & \\
\hline & Reg. subsidiado & 0.066 & 2.15 & 0.95 & 4.84 \\
\hline & No afiliado & 0.18 & 2.19 & 0.7 & 6.91 \\
\hline
\end{tabular}


Rocío Carvajal-Barona • LuIS Miguel Tovar-Cuevas •

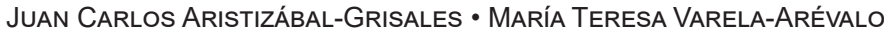

\begin{tabular}{|c|c|c|c|c|c|}
\hline \multicolumn{2}{|c|}{ Satisfacción de las necesidades del consumidor } & & & & \\
\hline \multirow{2}{*}{ Cuánto dura el tratamiento de TB } & Hasta seis meses & & 1 & & \\
\hline & Más de seis meses & 0 & $\underline{3.51}$ & 1.87 & 6.59 \\
\hline \multicolumn{6}{|c|}{ Características sistema de prestación servicios de salud } \\
\hline \multirow{2}{*}{$\begin{array}{l}\text { Tuvo problemas con el proveedor de } \\
\text { servicios de salud en el suministro } \\
\text { del tratamiento }\end{array}$} & No & & 1 & & \\
\hline & Sí & 0.028 & $\underline{\mathbf{5 . 5 3}}$ & 1.2 & 25.52 \\
\hline
\end{tabular}

Fuente: elaboración propia.

Los pacientes que refirieron no convivir con sus familiares (esposo(a), hijos, padres, hermanos) presentaron una oportunidad de incumplir el tratamiento. 4,54 veces mayor con relación a los pacientes que manifestaron convivir con sus familias.

De la misma manera, los pacientes que consideraban que sus relaciones familiares eran buenas presentaron una oportunidad $92 \%$ menor de incumplir el tratamiento, en comparación con los pacientes que informaron mantener malas relaciones familiares.

Con relación a la asistencia a reuniones de grupos religiosos o espirituales, aquellos pacientes que informaron que nunca asistían a dichas reuniones presentaron una oportunidad de incumplir el tratamiento 28.17 veces mayor que los que informaron que asistían diaria o casi diariamente a misa o culto.

Los pacientes que no han tenido que recibir tratamiento para otra enfermedad por más de dos semanas presentan una oportunidad 3.37 veces mayor de incumplir con el tratamiento que los que sí han tenido que recibir otros tratamientos por este tiempo.

Con respecto al consumo de drogas, de manera general (no durante el tratamiento), los pacientes que manifestaron haberlas consumido presentaron una oportunidad 3.41 veces mayor de incumplir con el tratamiento que los que no reportaron consumo de este tipo de sustancias.
Los pacientes que no reconocen que la duración del tratamiento de TB es hasta por seis meses presentaron una oportunidad de incumplir el tratamiento 3.51 veces mayor en comparación con los pacientes que manifestaron que este tratamiento dura hasta seis meses.

En relación con el tipo de afiliación, se debe tener en cuenta que aun cuando en este modelo multivariado dicha variable no consigue significancia estadística menor de $5 \%$, esta se encuentra muy cercana, con $6.6 \%$ para el caso del régimen subsidiado, es decir, que los paciente con afiliación al Sistema General de Seguridad Social en Salud (SGSSS) del régimen subsidiado presentaron una oportunidad de incumplir el tratamiento 2.15 veces mayor que los pacientes afiliados al SGSSS a través del régimen contributivo.

Finalmente y con relación al suministro del tratamiento por parte del servicio de salud, los pacientes que informaron falta de suministro presentaron una oportunidad 5.53 veces mayor de incumplir el tratamiento, en comparación con los pacientes que sí recibieron el tratamiento de manera ininterrumpida.

\section{Discusión}

Este estudio, realizado en dos ciudades de Colombia en las que se presenta un importante problema de XDR y MDR, en gran 
medida por dificultades para asegurar la adherencia al tratamiento anti-TB, encontró evidencia, a partir del análisis bivariado, de quince factores que se asocian con la adherencia al tratamiento anti-TB en Cali y Buenaventura. Sin embargo, el análisis multivariado solo ratificó la asociación con ocho de estos factores. Estos ocho factores, según el modelo de Aday y Andersen, se relacionan con: i) características de la población en situación de riesgo, como son: haber recibido tratamiento para otra enfermedad por más de dos semanas, tener buenas relaciones familiares, no vivir con la familia, no asistir a grupos religiosos, consumir drogas; ii) $l a$ satisfacción de las necesidades del consumidor: conocimiento de la duración del tratamiento; y iii) características del sistema de prestación de servicios de salud: falta de suministro ocasional del tratamiento.

Los otros factores que mostraron asociación significativa en el análisis bivariado fueron: estado civil, consumo de tabaco, ocupación, afiliación al sistema de salud, falta de recursos para asumir costos de transporte, antecedentes de complicaciones con el tratamiento de TB, haber tenido contacto (previo a la enfermedad) con personas con TB y creencias sobre la efectividad del tratamiento.

Las variables consideradas en este estudio han sido identificadas en revisiones sobre el tema $(5,19)$ y otras investigaciones conducidas en varios países como Colombia (11,20,21), Perú (22), Brasil (23,24), Argentina (10, 25), Chile (26), Rusia (27) y Etiopía (28), como factores que afectan la adherencia al tratamiento anti-TB. Sin embargo, los resultados parecen depender, en gran medida, de los diferentes contextos y de las características específicas de las poblaciones estudiadas. La evidencia sobre los factores asociados con la adherencia al tratamiento de TB entre países, e incluso entre regiones de un mismo país, en algunos casos es estadísticamente significativa y en otros no lo es.

Respecto a las variables relacionadas con factores personales como la tenencia de una red de apoyo social, expresada en el hecho de vivir con la familia o tener buenas relaciones familiares, se encuentran trabajos que apoyan los resultados de este estudio (20) y otros que no encuentran asociación con estos factores $(21,23)$. El consumo de drogas también ha sido identificado por varios estudios como un factor que incrementa el riesgo de no adherencia al tratamiento anti-TB $(5,22,26,27)$.

Variables como tener antecedentes de haber recibido otro tratamiento de larga duración en el pasado, así como otras variables relacionadas con la red de apoyo social, que además podrían relacionarse con la posibilidad de que los individuos sean más proclives a cumplir con rutinas como las que requiere el tratamiento DOTS-TAES, han sido menos exploradas en otras investigaciones. De acuerdo con algunos estudios, los aspectos psicosociales, expresados para este estudio en débiles redes de apoyo social y no experiencia previa en el manejo de tratamientos mayores a dos semanas, tienen un gran significado para la no adherencia en tratamientos de larga duración $(17,29)$.

Otro factor personal que incrementa el riesgo de no adherencia, y que se relaciona con los medios disponibles o capacidad de los individuos para acceder al tratamiento anti $\mathrm{TB}$, es la pertenencia al régimen subsidiado de salud que por definición es aquel al que pertenece la población colombiana más pobre. Resultados de otras investigaciones realizadas en Colombia no han encontrado asociación con esta variable (21), mientras que otros autores han encontrado que no tener acceso al plan obligatorio de servicios 
de salud de Colombia (POS) es un factor que incrementa el riesgo de no adherencia.

Si se considera que el acceso al POS está estrechamente ligado con el régimen de salud al que se pertenece y con la afiliación o no al sistema de salud, y que los regímenes pueden reflejar características tanto de la capacidad económica de los individuos como de la prestación de los servicios de salud, futuras investigaciones deberían profundizar para intentar establecer si el tipo de afiliación a salud es un factor determinante del abandono terapéutico, en cuanto que refleja, o bien la capacidad económica, o bien la ineficiencia del sistema de salud.

Respecto a los factores relacionados con la satisfacción de las necesidades del consumidor, la información que los pacientes tienen sobre el tratamiento anti-TB, como, por ejemplo, el tiempo que dura el tratamiento, también ha sido documentada como un factor que está asociado a la adherencia en otros estudios $(5,11)$.

En relación con las características del sistema de prestación de servicios de salud, la falta de suministro ocasional del tratamiento se identificó como factor de riesgo para la no adherencia al tratamiento anti-TB. Otros autores no han encontrado asociación estadísticamente significativa (21).

Barreras administrativas como la interrupción del suministro de medicamentos y la afiliación al régimen subsidiado de salud o régimen con menor capacidad de pago, se presentaron en este estudio, en particular, como factores de riesgo para la no adherencia al tratamiento de TB, lo cual nos indica que problemas de gestión administrativa y la ineficiencia del sistema de salud están disminuyendo la probabilidad de un control exitoso de la enfermedad en esta región de Colombia (17,30-33).
En general, los estudios sobre las barreras de adherencia a la terapia de TB se han centrado en el análisis de aspectos individuales e interpersonales del paciente, permitiendo la identificación de barreras asociadas al nivel cultural y educativo, por ejemplo.

Este estudio en comparación con otros similares tiene la ventaja de que no solo considera los posibles factores individuales e interpersonales que pueden afectar la adherencia al tratamiento de TB, sino que también explora el papel que otros factores de tipo social y administrativo pueden desempeñar en la adherencia a dicho tratamiento. Entre los sesgos potenciales del estudio se destaca que puede existir sesgo de memoria, pues la investigación fue retrospectiva e indagó por un evento sucedido entre seis meses y un año antes.

Los resultados de este estudio aportan no solo al conocimiento de las barreras para la terapia medicamentosa de TB en Cali y Buenaventura, sino también al replanteamiento de las estrategias utilizadas hasta el momento en los programas de control en esta región colombiana, centradas en actividades asistenciales y comportamentales del paciente frente al tratamiento. Los resultados señalan la necesidad de ir consolidando estrategias transectoriales e interdisciplinares que permitan abarcar los otros factores administrativos y sociales que afectan la adherencia al tratamiento de TB.

\section{Agradecimientos}

$\mathrm{Al}$ Fondo mundial de lucha contra el sida, la tuberculosis y la malaria, y a la Organización Internacional para las Migraciones de Colombia, por la financiación de esta investigación. A las secretarías de Salud de Cali y Buenaventura y a la Secretaría de Salud Departamental del Valle del Cuaca. A los expacientes de TB y a sus familias de Cali y 
Buenaventura por la participación en este estudio. A la Asociación Nacional de Alcaldes y Gobernadores de Municipios y Gobernaciones con Población Afrodescendiente(Amunafro) por el apoyo para el desarrollo local del proyecto de investigación.

\section{Referencias bibliográficas}

1. World Health Organization (WHO). Global tuberculosis report 2015, $20^{\mathrm{Th}}$ edition. Ginebra: WHO; 2015.

2. World Health Organization (WHO). Global tuberculosis control: WHO report 2011. Ginebra: WHO; 2011.

3. Lönnroth K, Jaramillo E, Williams B, Dye C, Raviglione M. Tuberculosis: the role of risk factors and social determinants. En: World Heatlh Organization. Equity, social determinants and public health programmes. Ginebra: WHO; 2010.

4. Rondags A, Himawan AB, Metsemakers J, Kristina TN. Factors influencing non-adherence to tuberculosis treatment in Jepara, Central Java, Indonesia. Southeast Asian J Trop Med Public Health. 2014;45(4):859-67.

5. Cáceres FM. Factores de riesgo para abandono (no adherencia) del tratamiento antituberculoso. Med UNAB. 2004;7(21):172-80.

6. Vijay S, Kumar P, Chauhan LS, Vollepore BH, Kizhakkethil UP, Rao SG. Risk factors associated with default among new smear positive TB patients treated under DOTS in India. PLOS ONE. 2010;5(4): e10043. doi:10.1371/journal.pone.0010043

7. Chaudhry LA, Zamzami M, Aldin S, Pazdirek J. Clinical consequences of non-compliance with directly observed therapy short course (DOTS): Story of a recurrent defaulter. International Journal of Mycobacteriology. 2012;(1):99-103.

8. Dueñes M, Cardona D. Factores relacionados con la adherencia al tratamiento en pacientes con tuberculosis. Pereira, Colombia 2012-2013. Biomédica. 2016;36(3).

9. Torres Z, Herrera T. Perfil del paciente con tuberculosis que abandona el tratamiento en Chile. Revista Chilena Enfermedad Respiratoria. 2015;(31):52-7.

10. Herrero MB, Ramos S, Arrossi S. Determinants of non-adherence to tuberculosis treatment in Argentina: barriers related to access to treatment. Rev Bras Epidemiol. 2015;18(2):287-98. doi:10.1590/19805497201500020001

11. Navarro-Quintero C, Rueda Rincón JL, Mendoza Ojeda JL. Factores asociados al abandono del tratamiento antituberculoso en pacientes con TB. Revista Ciencia y Cuidado. 2013;10(1):19-27.

12. Bagchi S, Ambe G, Sathiakumar N. Determinants of poor adherence to anti-tuberculosis treatment in Mumbai, India. Int J Prev Med. 2010;1(4):223-32.
13. Mishra P, Hansen EH, Sabroe S, Kafle KK. Adherence is associated with the quality of professional-patient interaction in Directly Observed Treatment Short-course, DOTS. Patient Education and Counseling. 2006;63: 9-37.

14. República de Colombia, Departamento Administrativo Nacional de Estadística (DANE). Censo General de Población 2005. Bogotá: DANE; 2005.

15. Gobernación del Valle del Cauca, Secretaría de Salud Departamental. Plan estratégico Valle del Cauca Libre de Tuberculosis 2010-2015, para la expansión y fortalecimiento de la estrategia alto a la TB, segunda edición. Cali: Gobernación del Valle del Cauca; 2013.

16. Resolución 008430 de 1993 (4 de octubre de 1993), República de Colombia, Ministerio de Salud.

17. Mateus-Solarte JC, Carvajal-Barona R. Factors predictive of adherence to tuberculosis treatment, Valle del Cauca, Colombia. INT J Tuberc Lung Dis. 2008;12(5):520-6.

18. Aday LA, Andersen R. A framework for the study of access to medical care. Health Services Research. 1974;9(3):208-20.

19. Arrossi S, Herrero MB, Greco A, Ramos S. Factores asociados a la no-adherencia al tratamiento de la tuberculosis: revisión de la literatura. Buenos Aires: Centro de Estudios de Estado y Sociedad; 2011.

20. Cáceres FM, Orozco LC. Incidencia y factores asociados al abandono del tratamiento antituberculoso. Biomédica. 2007;27:498-504.

21. Rodríguez-Alviz E, Mondragón CH. Adherencia a la terapia farmacológica y sus factores determinantes en pacientes con tuberculosis de un centro de salud de Santiago de Cali. Rev Colomb Cienc Quím Farm. 2014; 43(1):104-19.

22. Culqui DR, Grijalva CG, Reategui SR, Cajo JM, Suárez LA. Factores pronósticos del abandono del tratamiento antituberculoso en una región endémica del Perú. Rev Panam Salud Pública. 2005;18(1):14-20.

23. Paixao LM, Gontijo ED. Perfil de casos de tuberculose notificados e fatores associados ao abandono, Belo Horizonte, MG. Rev Sáude Pública. 2007; 41(2):205-13.

24. Albuquerque MFPM, Ximenes RAA, Lucena-Silva N, Souza WV, Dantas AT, Dantas OMS, et al. Factors associated with treatment failure, dropout, and death in a cohort of tuberculosis patients in Recife, Pernambuco State, Brazil. Cad Saúde Pública. 2007;23(7):1573-82.

25. Herrero MB, Arrossi S, Ramos S, Braga JU. Análisis espacial del abandono del tratamiento de tuberculosis, Buenos Aires, Argentina. Rev. Saúde Pública. 2015;(49).

26. Torres Z, Herrera T. Perfil de pacientes con tuberculosis que abandona el tratamiento en Chile. Rev Chil Enf Respir. 2015;(31):52-7.

27. Gelmanova IY, Keshavjee S, Golubchikova VT, Berezina VI, Strelis AK, Yanova GV, Atwoodd S, Murray M. Barriers to successful tuberculosis treatment in Tomsk, Russian Federation: non-adherence, default and the acquisition of multidrug resistance. Bulletin of the World Health Organization. 2007;85(9):703-11. 


\section{Rocío CARVAJAL-BARONA • LUIS MIGUEL TOVAR-CuEVAS •

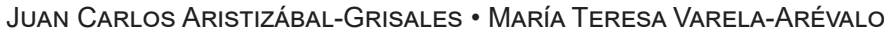

28. Shargie EB, Lindtjørn B. Determinants of treatment adherence among smear positive pulmonary tuberculosis patients in southern Ethiopia. PLoS Med. 2007;4(2).

29. World Health Organization (WHO). Adherence to long-term therapies. Evidence for action. Ginebra: WHO; 2003.

30. Carvajal R, Cabrera G, Mateus J. Efectos de la reforma en salud en las acciones de control de tuberculosis en el Valle del Cauca, Colombia. Biomédica. 2004; 24(Supl.):138-48.

31. Ayala-Cerna C, Kroeger A. La reforma del sector salud en Colombia y sus efectos en los programas de control de tuberculosis e inmunización. Cad Saúde Pública. 2002; 18(6):1771-81.

32. Hernández A, Vega R. El Sistema Colombiano de Seguridad Social en Salud: desigualdad y exclusión. Gerencia y Políticas de Salud. 2012;1(1):48-73.

33. Laserson KF, Osorio L, Sheppard JD. Et. Clinical and programmatic mismanagement rather than community outbreak as the cause of chronic, drug-resistant tuberculosis in Buenaventura, Colombia 1988. The International Journal of Tuberculosis and Lung Disease. 2000;4(7):673-83. 